\title{
ON SEPARABILITY IN LINEAR TOPOLOGICAL SPACES
}

\author{
ROBERT H. LOHMAN AND WILBUR J. STILES
}

\begin{abstract}
This paper contains (1) an example of a separable linear topological space with a closed nonseparable linear subspace, and (2) a proof of the fact that every metrizable subspace of a separable linear topological space is separable.
\end{abstract}

It is well known that every subspace of a separable metric space is separable, and it is also well known that there are separable topological spaces with nonseparable subspaces. In view of this, it seems natural to ask if every (closed) linear subspace of a separable linear topological space is separable. The purpose of this short note is to consider this question, and specifically (1) to give an example of a separable linear topological space with a closed nonseparable linear subspace, and (2) to show that any metrizable linear subspace of a separable linear topological space is separable.

It is not difficult to give an example of a separable linear topological space $X$ with a (dense) nonseparable subspace $Y$. In fact, the product of continuum many lines with the usual product topology can be taken for $X$ when $Y$ is the subspace consisting of all functions which vanish on all but finitely many points of the indexing set. The problem of finding a separable space with a closed nonseparable subspace seems to be more difficult.

EXAmple. Let $X$ be the conjugate space of $l^{\infty}$ where $l^{\infty}$ is the space of all bounded sequences $\left(x_{n}\right)$ with $\left\|\left(x_{n}\right)\right\|=\sup \left|x_{n}\right|$, and let $X$ have its weak-star topology. H. P. Rosenthal has shown [1, Proposition 3.4] that $X$ contains an isomorphic copy $Y$ of a nonseparable Hilbert space $H$. Since $Y$ is reflexive, $Y$ is a weak-star closed subspace of $X$ by Proposition 1.2 of [1]. Since $Y$ is weak-star closed, $Y$ is isometrically isomorphic to $\left(l^{\infty} / Y^{\perp}\right)^{*}$. The reflexivity of $Y$ and the standard duality arguments imply that the restriction of the weak-star topology to $Y$ is the same as the weak topology of $Y$. Since $Y$ is not separable, $Y$ is not weakly separable. Theref ore, $Y$ is not weak-star separable because the restriction of the weak-star topology to $Y$ is the same as the weak topology of $Y$.

Received by the editors June 13, 1972 and, in revised form, November 10, 1972.

AMS (MOS) subject classifications (1970). Primary 46A15; Secondary 57A17.

Key words and phrases. Separable linear space, nonseparable linear subspace, metrizable linear subspace.

(c) American Mathematical Society 1974 
We conclude by giving a condition which is sufficient to insure that a subspace of a separable linear topological space is separable.

THEOREM. Let $E$ be a separable linear topological space and $F$ a semimetrizable linear subspace of $E$. Then $F$ is separable.

Proof. Since $F$ is semimetrizable there is a countable collection of neighborhoods $\left\{M_{k}\right\}$ of 0 in $E$ such that $\left\{M_{k} \cap F\right\}$ is a local base for the topology of $F$. Choose a countable collection $\left\{N_{k}\right\}$ of balanced neighborhoods of 0 in $E$ inductively as follows. Choose $N_{1}$ such that $N_{1} \subset M_{1}$. Having chosen $N_{n}$, choose $N_{n+1}$ such that $N_{n+1}+N_{n+1} \subset N_{n}$ and $N_{n+1} \subset$ $M_{n+1}$. By a well-known theorem of Kakutani, the collection $\left\{N_{k}\right\}$ is the local base for a linear semimetric topology $\tau$ for $E$. Clearly $\tau$ restricted to $F$ is the original topology of $F$. Since $E$ is separable and $\tau$ is weaker than the original topology, $E$ is separable with $\tau$. This, of course, implies that the subspace $F$ is separable.

\section{REFERENCE}

1. H. P. Rosenthal, On quasi-complemented subspaces of Banach spaces, with an appendix on compactness of operators from $L^{p}(\mu)$ to $L^{r}(v)$, J. Functional Analysis 4 (1969), 176-214. MR 40 \#3277.

Department of Mathematics, Kent State University, Kent, Ohio 44242

Department of Mathematics, Florida State University, Tallahassee, Florida 32306 